\title{
НЕВРОЛОГИЧЕСКИЕ АСПЕКТЫ COVID-19 И ЕГО ВЛИЯНИЕ НА ФОРМИРОВАНИЕ ДЕМЕНЦИИ У ПОЖИЛЫХ ЛЮДЕЙ
}

\section{NEUROLOGICAL ASPECTS OF COVID-19 AND ITS IMPACT ON THE FORMATION OF DEMENTIA IN THE ELDERLY}

\section{E. Vishneva}

N. Vesnina

Summary. Coronavirus infection (COVID-19) caused by Coronavirus 2 Severe Acute Respiratory Syndrome (SARS-CoV-2) has spread rapidly around the world and has had an unprecedented impact on health systems, economies and societies. The clinical picture of COVID-19 primarily affects the respiratory system, causing bilateral pneumonia, but the infection is increasingly recognized as a systemic disease, and neurological manifestations are noted not only in patients with mild symptoms, but more often in patients in serious condition. Elderly people are at high risk of developing severe forms of COVID-19 due to factors associated with aging of all body systems and a higher prevalence of comorbidities, and, therefore, they are more vulnerable to possible severe neuropsychiatric and cognitive impairments. Several cases described such manifestations as insomnia, depressed mood, anxiety, post-traumatic stress disorder and cognitive impairment in a subset of patients after hospital discharge. The potential mechanisms underlying these symptoms are not fully understood, but are likely multifactorial, including the direct neurotrophic effect of SARS-CoV-2, the consequences of prolonged stay in the intensive care unit, the use of mechanical ventilation and sedatives, brain hypoxia, systemic inflammation, secondary effects of drugs used to treat COVID-19 and peripheral organ dysfunction. Chronic diseases such as dementia are of particular concern, not only because they are associated with higher hospital admissions and mortality rates, but also because COVID-19 further exacerbates the vulnerability of people with cognitive impairments. In patients with dementia, COVID-19 often has an atypical picture with changes in mental status that make early detection of cases difficult. COVID-19 has had a huge impact on rehabilitation facilities, where infection and death rates are very high. Measures taken to slow the spread of the virus have forced social distancing and withdrawal of cognitive rehabilitation programs, which may have contributed to loneliness, behavioral symptoms, and cognitive decline in dementia patients. COVID-19 survivors should be periodically assessed using comprehensive cognitive and neuropsychiatric assessments, and special mental health and cognitive rehabilitation programs should be provided for those with delayed cognitive and psychiatric consequences.

Keywords: COVID-19, bilateral pneumonia, cognitive impairment, neurotrophic effect, dementia, mental status, cognitive rehabilitation, Alzheimer's disease.
Вишнева Елена Михайловна

Д.м.н., дочент, врач-кардиолог высшей категории, ФГБОУ ВО «Уральский Государственный Медицинский Университет», главный врач, сеть клиник ООО «Семейная клиника», г. Екатеринбург e.m.vishneva@mail.ru

Веснина Наталья Сергеевна Врач-невролог, ГАУЗ СО «Городская клиническая больница № 14», г. Екатеринбург vesninans@mail.ru

Аннотация. Коронавирусная инфекция (COVID-19), вызванная тяжелым острым респираторным синдромом коронавируса 2 (SARS-CoV-2), быстро распространилась по всему миру и оказала беспрецедентное воздействие на системы здравоохранения, экономику и общество. Клиническая картина COVID-19 в первую очередь влияет на дыхательную систему, вызывая двустороннюю пневмонию, однако инфекция все чаще признается системным заболеванием, при этом неврологические проявления отмечаются не только у пациентов с легкими симптомами, но чаще всего у пациентов в тяжелом состоянии. Пожилые люди подвержены высокому риску развития тяжелых форм COVID-19 из-за факторов, связанных со старением всех систем организма, и более высокой распространенности сопутствующих заболеваний, а, следовательно, они более уязвимы для возможных тяжелых психоневрологических и когнитивных нарушений. В нескольких наблюдениях описывались такие проявления, как бессонница, подавленное настроение, беспокойство, посттравматическое стрессовое расстройство и когнитивные нарушения у части пациентов после выписки из больницы. Потенциальные механизмы, лежащие в основе этих симптомов, полностью не изучены, но, вероятно, являются многофакторными, включая прямой нейротрофический эффект SARS-CoV-2, последствия длительного пребывания в отделении интенсивной терапии, использование искусственной вентиляции легких и седативных препаратов, гипоксию мозга, системное воспаление, вторичное эффекты лекарств, используемых для лечения COVID-19 и дисфункции периферических органов. Хронические заболевания, такие как деменция, вызывают особую озабоченность не только потому, что они связаны с более высокими показателями госпитализации и смертности, но и потому, что COVID-19 еще больше усугубляет уязвимость людей с когнитивными нарушениями. У пациентов с деменцией COVID-19 часто имеет нетипичную картину с изменениями психического статуса, затрудняющими раннее выявление случаев. COVID-19 оказал огромное влияние на реабилитационные учреждения, где уровень инфицирования и смертности очень высок. Меры, принятые для замедления распространения вируса, вынудили к социальному дистанцированию и отмене программ когнитивной реабилитации, что, возможно, способствовало возникновению одиночества, поведенческих симптомов и ухудшению когнитивных функций у пациентов с деменцией. Лица, пережившие COVID-19, должны периодически проходить оценку с помощью комплексных когнитивных и нейропсихиатрических оценок, а для тех, кто страдает отсроченными когнитивными и психиатрическими последствиями, долж- 


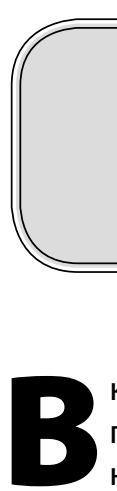

конце декабря 2019 года Китай сообщил о группе случаев пневмонии, вызванной новым коронавирусом, тяжелым острым респираторным синдромом коронавирусом 2 (SARS-CoV-2), названную в последующем коронавирусной болезнью 2019 (COVID-19). Инфекция SARS-CoV-2 в первую очередь поражает дыхательную систему, вызывая лихорадку и кашель - два из наиболее распространенных острых симптомов среди всех симптомов [1], около $20 \%$ людей могут страдать опасными для жизни респираторными осложнениями [2]. COVID-19 все чаще признается системным заболеванием, поражая другие системы органов. О множественных неврологических проявлениях сообщалось примерно в 35,6\% случаев [3]. Они включают неспецифические симптомы, в основном головную боль и миалгию, а также гипосмию и дисгевзию, но есть данные и о более серьезных осложнениях: нейровоспалительные синдромы, энцефалопатии, ишемические инсульты или синдром Гийена-Барре [4]. Эти неврологические проявления чаще наблюдаются у тяжелобольных пациентов [5]. В большой когорте из 841 пациента, госпитализированных из-за COVID-19 из двух клинических центров в Испании, неврологические симптомы присутствовали в 54,7\% случаев, у пациентов с тяжелой инфекцией в 64,7\% [6]. Измененные уровни сознания были наиболее частым неврологическим проявлением в этой же группе [6]. Легкие нарушения сознания наряду с очаговыми неврологическими нарушениями явились причиной обращения у 2,5\% пациентов, а неврологические осложнения стали основной причиной смерти у $4,1 \%$ от общего числа умерших.

Пандемия в большей степени затрагивает пожилое население. Уровень госпитализации и смертности резко возрастает после 65 лет [7]. Текущие данные указывают на пожилой возраст, а также на мужской пол и наличие сопутствующих заболеваний, как факторов плохого прогноза и более высокий риск смерти [8]. Более того, у пожилых людей с хроническими заболеваниями, такими как деменция, в начале заболевания COVID-19 часто наблюдаются атипичные симптомы, такие как изменение психического статуса (включая спутанность сознания, возбуждение, дезориентацию, отказ от помощи, потерю аппетита) [9]. Такое атипичное проявление может отсрочить своевременный диагноз и лечение и, следовательно, ухудшить прогноз и выживаемость. ны быть предусмотрены специальные программы психического здоровья и когнитивной реабилитации.

Ключевые слова: COVID-19, двусторонняя пневмония, когнитивные нарушения, нейротрофический эффект, деменция, психический статус, когнитивная реабилитация, болезнь Альцгеймера.

Таким образом, пожилые люди не только чаще страдают более тяжелым течением заболевания COVID-19, но и более уязвимы для стойкого формирования инвалидизации. Осложнения у выживших пациентов в настоящее время неизвестны. Однако, как это было замечено при аналогичной вирусной инфекции и выживших после критического состояния заболевания, у некоторых из этих пациентов в ближайшие месяцы и годы могут появиться неврологические последствия в виде стойких психоневрологических и когнитивных нарушений [10]. Бессонница, тревога, симптомы посттравматического стресса, психоз и расстройства настроения, описаны в нескольких источниках $[11,12,13,14,6,15]$. Исследование, в ходе которого были получены данные с глобальной базы, в которую вошли медицинские записи о 40469 случаях заражения COVID-19, в основном из Соединенных Штатов (США) (76\%), показало, что 22,5\% имели неврологические и / или психиатрические проявления и тревожные расстройства [13].

Наблюдательное исследование в Великобритании показало, что 39 случаев из когорты из 125 госпитализированных пациентов с COVID-19 с неврологическими проявлениями имели измененный психический статус, 16 с энцефалопатией и 23 с нервно-психическими синдромами, в основном с впервые возникшими психозами $(\mathrm{n}=10)$ или другими связанными психическими расстройствами ( $\mathrm{n}=7)$ [16]. В соответствии с этими доказательствами, в ретроспективном описательном исследовании, проведенном в больнице в Мадриде, Испания, 10 пациентов с лабораторно подтвержденным диагнозом COVID-19 и впервые появившимися психотическими симптомами были выявлены среди 10000 пациентов с симптомами, совместимыми с оценкой COVID-19, с марта по апрель 2020 года в отделении неотложной помощи [17]. Их средний возраст составлял 54,1 года, психиатрические симптомы включали бред, нарушения ориентации в месте и времени, нарушение внимания и слуховые галлюцинации (в 10, 6 и 4 случаях соответственно), данные симптомы появились в основном после первых типичных симптомов COVID-19 и разрешились в течение менее 2 недель [17]. Эти эпизоды считались атипичными, поскольку пациенты не имели в анамнезе ранее психических расстройств, расстройств, связанных с употреблением психоактивных веществ, имели атипичный возраст начала и быстро выздоравливали [17]. Авторы предположили, что эти атипичные психотические эпизо- 
ды могут быть объяснены системными воспалительными реакциями, основанными на результатах аналитических и дополнительных тестов, или побочными эффектами, связанными с лечением COVID-19 [17].

Тяжелобольные пациенты с COVID-19, которым требуется госпитализация в отделение интенсивной терапии (ОИТ), также наиболее подвержены риску развития делирия, тяжелой деменции, которые еще больше усугубляются частой потребностью в высоких дозах седативных средств, пожилым возрастом и наличием множественных сопутствующих заболеваний [18]. В обсервационном исследовании, проведенном во Франции, 40 из 58 (69\%) пациентов с COVID-19, посещенных в отделении интенсивной терапии, демонстрировали ажитацию, а у 26 из них было выявлено состояние спутанности сознания [19]. Нейровизуализация головного мозга выявила двустороннюю лобно-височную гипоперфузию у 11 пациентов и большие лептоменингеальные пространства у 8 из них [19].

Также имеются данные о преобладании депрессивных симптомов у тех, кто уже вылечился от COVID-19 [20, 21, 22]. Исследование 126 выживших после COVID-19 выздоравливающих из Шэньчжэня, Китай, показало, что тревога и депрессия, о которых сообщали сами пациенты, были обычным явлением после выписки из больницы [20], и, кроме того, депрессивные симптомы были связаны с подавлением иммунной системы, основанным на увеличении количества лейкоцитов и воспалительных факторов [21].

Несмотря на эти предварительные данные, большинство результатов было получено на основе самооценки шкал без клинических и диагностических оценок, т.о., необходимы дальнейшие обследования и последующие наблюдения больных, чтобы определить не только то, связаны ли эти симптомы с самой инфекцией, вторичными иммунными реакциями, побочными действиями лечения или психологическими стрессами, но также, улучшаются, сохраняются или ухудшаются они с течением времени.

Существует очень мало исследований, сообщающих о когнитивных симптомах, связанных с COVID-19. Данные 431051 участника проспективного исследования в Великобритании показывают, что несколько психосоциальных факторов были связаны с риском госпитализации из-за COVID-19, но после учета других значимых переменных (социально-демографические, социально-экономические, психологические факторы, факторы образа жизни и сопутствующие медицинские заболевания), единственным значимым фактором, связанным с риском заражения, была изначально более низкая когнитивная функция [23]. В ретроспективном исследовании, проведенном в Чикаго, США, среди 50 госпитализированных пациентов c COVID-19, которые поступили в неврологическое отделение или имели неврологические симптомы, 24\% из них имели кратковременную потерю памяти [24]. Наблюдательное исследование в Великобритании показало, что 6 случаев из когорты из 125 госпитализированных пациентов с COVID-19 с неврологическими проявлениями имели нейрокогнитивное расстройство [16].

Также есть предварительные наблюдения когнитивных нарушений после выписки из больницы. В этом ключе в обсервационном исследовании, проведенном во Франции, более одной трети (15/45) пациентов продемонстрировали когнитивные нарушения при выписке из отделения интенсивной терапии, особенно в форме дизэкспективного синдрома, характеризующегося невнимательностью, дезориентацией и плохо организованными движениями в ответ на команды [19]. В серии случаев из 4 пациентов с тяжелой формой COVID-19, которым потребовалась госпитализация в ОИТ, когнитивные нарушения, идентифицированные как дефицит памяти и фронтальный синдром, были обнаружены после выписки, но были восстановлены через 5 дней терапии иммуноглобулином [25]. Кроме того, в выборке из 71 госпитализированного пациента с COVID-19 те, у кого был диагностирован делирий во время госпитализации (42\%), имели более низкие когнитивные показатели при телефонном скрининговом собеседовании в течение 4 недель после выписки, хотя межгрупповое сравнение не дало результатов статистической значимости $(p=0,06)$ [26].

Отсутствие более точной информации о когнитивных симптомах у пациентов с COVID-19 можно объяснить влиянием пандемии на системы здравоохранения, а также, в тяжелых случаях, трудностью проведения комплексной нейропсихологической оценки. Однако эта информация будет иметь большое значение для выявления факторов риска, связанных с острыми когнитивными симптомами, связанными с этим заболеванием, как у людей с предшествующими когнитивными нарушениями, так и без них, а также для того, чтобы пролить свет на лежащие в их основе механизмы. Также необходимо предложить нейропсихологическую реабилитацию тем, кто в ней нуждается. Важно и безотлагательно свести к минимуму потенциальное негативное воздействие на когнитивные и психосоциальные функции, а так же качество жизни.

Отсроченные осложнения у тех пациентов, которые пережили болезнь, в настоящее время неизвестны, но ожидается, что они появятся в ближайшие месяцы и годы, как это наблюдалось в прошлых пандемиях, вызванных гриппом или аналогичными коронавирусами, такими как MERS-CoV и SARS-CoV [14, 27], а также у переживших критическое состояние, которым требовалась поддержка в отделении интенсивной терапии $[28,29,30]$. 
В систематическом обзоре и метаанализе, проведенном [14] в отношении острых нейропсихиатрических проявлений коронавирусных инфекций и после перенесенных болезней было оценено 72 исследования, включая SARS-CoV ( $n=47)$, MERS-CoV $(n=13)$ и текущий SARSCoV-2 ( $n=12)$, возраст госпитализированных пациентов колебался от 12 до 68 лет [14]. SARS-Cov и MERS-CoV были связаны с преобладающими нейропсихиатрическими симптомами как в острой фазе, так и после выздоровления [14]. Результаты проведенного метаанализа показали, что после выздоровления расчетная распространенность посттравматического стрессового расстройства составляла 32,2\% (средний период наблюдения 33,6 месяца), а распространенность тревожных и депрессивных расстройств составляла 15\% (средний период наблюдения 11,6 и 22,6 месяца) соответственно [14]. Показатели качества жизни были значительно ниже у пациентов по сравнению с контрольной группой, и 76,9\% вернулись к работе в среднем через 3 года наблюдения [14]. Результаты одного из исследований, включенных в этот обзор, с выборкой из 181 человека, инфицированного SARS-CoV-1, показали, что, хотя только 3,3\% пациентов имели в анамнезе психические расстройства до вирусной инфекции, после среднего периода наблюдения в 3,4 года 42,5\% соответствовали клиническим критериям как минимум одного психического заболевания, при этом посттравматическое стрессовое расстройство было наиболее распространенным расстройством (54,5\%), за которым следовала депрессия (39\%) [10]. Аналогичным образом, у 70,8\% подтвержденных случаев SARSCoV развились психиатрические симптомы, и 40\% из них были поставлены во время госпитализации, в то время как ни один из подозреваемых случаев, которые были помещены в карантин, но дал отрицательный результат теста на вирус, не продемонстрировал каких-либо психиатрических симптомов [31]. Таким образом, эти данные свидетельствуют о возможной роли коронавирусных инфекций в индукции изменений мозга, связанных с этими психиатрическими симптомами.

Кроме того, госпитализация в ОИТ и инвазивные методы лечения, такие как вентиляция и седация, после острого респираторного дистресс-синдрома (ОРДС) являются факторами риска снижения когнитивных функций [32]. В рамках текущей пандемии, вызванной SARS-CoV-2, ретроспективное исследование серии случаев с участием 1591 пациента с COVID-19, поступивших в отделение интенсивной терапии в Италии, показало, что 88\% нуждались в механической вентиляции легких [33], а в меньшем исследовании, проведенном в отделении интенсивной терапии в Вашингтоне, США, у 71\% из 21 тяжелобольного пациента развился ОРДС и потребовалась искусственная вентиляция легких [34]. Данные об отдаленных результатах у взрослых, которым требовалась искусственная вентиляция легких, показали когнитивные нарушения внимания, памяти, беглости речи, скорости обработки информации или управляющих функций у 78 и 47\% пациентов после 1 и 2 лет выписки соответственно [35,36]. В общей сложности 15 пациентов в этом исследовании также прошли томографию головного мозга, и, по сравнению с контрольной группой того же возраста и пола, у них были значительно большие объемы желудочков мозга [36]. Даже после 5 лет наблюдения у 20\% выживших после ОРДС наблюдались когнитивные последствия в самых разных когнитивных областях [29].

Основные причины этих симптомов, связанных c COVID-19, и механизмы, участвующие в нарушениях, в настоящее время полностью не изучены, но, вероятно, имеют многофакторный характер. Эти факторы включают прямую вирусную инфекцию нервной системы, системную воспалительную реакцию, цереброваскулярную ишемию из-за эндотелиальной дисфункции или тяжелой коагулопатии, ОРДС, проявляющийся в тяжелых случаях, использование инвазивной вентиляции и седативных средств наряду с побочными эффектами применяемых лекарственных средств для лечения COVID-19 и дисфункции периферических органов [32, 37, 38].

SARS-CoV-2, как и другие коронавирусы, проявляет определенный нейротропизм. Были выдвинуты два возможных пути внутричерепного распространения коронавирусов: прямая гематогенная атака и ретроградное восхождение через периферические нервные волокна верхних дыхательных путей [39]. SARS-CoV-2 использует белки SPIKE, расположенные на его поверхности, для связывания рецептора ангиотензинпревращающего фермента 2 на клетках-хозяевах млекопитающих [40]. Было обнаружено, что рецептор АПФ2 широко экспрессируется в нейронах и глиальных клетках [41]. Имеются доказательства гибели нейронов после заражения мозга SARS-CoV-1 через обонятельную луковицу в моделях на животных [42].

На сегодняшний день существует мало свидетельств прямого воздействия инфекции на мозг, связанной c COVID-19. Результаты анализов полимеразной цепной реакции с обратной транскрипцией образцов спинномозговой жидкости (ЦСЖ), проведенные в нескольких случаях COVID-19 с неврологическими проявлениями, были отрицательными для SARS-CoV-2 [25,29].

Доступны немногочисленные нейропатологические данные о случаях COVID-19, которые в основном показывают гипоксические изменения и демиелинизирующие поражения [43, 44, 45]. Серия патологоанатомических вскрытий, проведенная в Германии, выявила вирусную нагрузку SARS-CoV-2 в головном мозге, почках, печени, сердце и крови [46]. Необходимы дальнейшие исследования, чтобы определить, вызваны ли эти поражения ин- 
фекцией SARS-CoV-2 или вторичными состояниями, связанными с болезнью. Недавний систематический обзор, который включал 26 исследований нейровизуализации, большинство из которых были сериями случаев пациентов с COVID-19, которые прошли визуализацию головного мозга из-за неврологических симптомов, обнаружил что в 34\% (124 из 361) случаев наблюдались поражения головного мозга, вероятно, связанные с COVID-19, и среди них наиболее частой находкой были диффузные подкорковые аномалии и аномалии глубокого белого вещества. Другими частыми находками, хотя и менее распространенными, были микрокровоизлияния, кровоизлияния и инфаркты [47].

Даже при отсутствии прямой инфекции головного мозга тяжелая системная реакция также может быть связана с ускорением нейровоспалительных реакций, которые могут способствовать последующему повреждению ткани мозга $[48,49,50]$. В тяжелых случаях вирус может вызвать усиленный и дисрегулируемый ответ организма, так называемый «цитокиновый шторм», который включает в себя повышенные уровни провоспалительных цитокинов, таких как фактор некроза опухоли (TNF) и интерлейкин-6 (IL-6). Если этот ответ сохраняется в течение долгого времени, он создает состояние системного воспаления, что приводит к нарушению гематоэнцефалического барьера и повреждению нервных и глиальных клеток, что может быть связано с формированием отсроченных последствий у выживших. Текущие данные, относящиеся к SARS-CoV-2, показывают, что обычно у наиболее сильно пораженных пациентов наблюдается повышенный уровень провоспалительных цитокинов $[51,52,53]$.

Хроническое системное воспаление также изучалось как один из основных патогенетических механизмов, вовлеченных в нейродегенеративное заболевание, такое как болезнь Альцгеймера (БА) [54]. В выборке из 12336 участников со средним возрастом 56,8 года системное воспаление было изучено с использованием совокупной оценки биомаркеров крови, и результаты показали значительную связь между исходным воспалением и ускоренным снижением когнитивных функций после последующего наблюдения в течение 20 лет [55]. Воспаление, связанное с вирусной инфекцией, значительно ухудшает патологию, связанную с тау-белком, и приводит к ухудшению памяти [56]. Гиппокамп, область, участвующая в формировании памяти, особенно уязвима для респираторных вирусных инфекций [57]. Кратковременное ухудшение зависимого от гиппокампа обучения и снижение долгосрочной потенциации, связанное с нарушением пространственной памяти, наблюдались у мышей, инфицированных вирусом гриппа [58]. Кроме того, в присутствии провоспалительных цитокинов микроглиальные клетки теряют свою способность фагоцитировать $\beta$-амилоид, что может быть связано с накоплением амилоидных бляшек, одним из отличительных признаков БА [59].

Было обнаружено, что аллель гена APOE-ع4, самый сильный генетический фактор риска БА, связан с повышенным риском инфекции и смертности из-за COVID-19, хотя биологические механизмы, участвующие в этой ассоциации, остаются неизвестными [60]. Однако, как сообщается, экспрессия АПФ2 снижается в средней части лобной ткани головного мозга у пациентов с БА, особенно у пациентов, несущих аллель APOE $\varepsilon 4$, и это снижение отрицательно коррелирует с патологией А $\beta$ и фосфорилированного тау-белка [61]. Таким образом, вирусная инфекция может быть отягчающим фактором нейродегенерации у людей с чувствительными генетическими вариантами.

Несмотря на эти предварительные доказательства, связанные с COVID-19, необходимы дальнейшие исследования, чтобы установить основные причины этих симптомов и механизмы, участвующие в потенциальных длительных нарушениях, а также, может ли этот коронавирус вызывать или обострять нейродегенеративные заболевания.

У пожилых людей с хроническими заболеваниями, такими как деменция, развиваются более серьезные и часто летальные формы COVID-19 [9, 62, 63]. Люди с деменцией, скорее всего, не смогут следовать рекомендациям по снижению передачи вируса (таким как самоизоляция, соблюдение дистанции, частое мытье рук и использование масок для лица) [64], т.о., формируя более высокую вероятность заражения. Обзор 627 пациентов, госпитализированных в отделение неотложной медицинской помощи с пневмонией COVID-19 в Италии, показал, что у пациентов с деменцией ( $\mathrm{n}=$ $82,13,1 \%$ ) уровень смертности был значительно выше, чем у пациентов с когнитивными нарушениями $(62,2 \%$ против 26,2\%, p <0,001) [9]. В самой большой популяции в исследовании, проведенном на сегодняшний день и включающем 17278392 вероятных случаев COVID-19 из Англии, предыдущий анамнез инсульта или деменции был связан с повышенным риском смерти через 90 дней [2,16 65]. Точно так же в учреждении ухода, расположенном в Вашингтоне, США, среди 101 жителя, положительного на COVID-19, коэффициент летальности был чрезвычайно высоким (33,7\%) [66]. Следует принять во внимание то, что, учитывая быстрое развитие случаев COVID-19, которые требовали медицинской помощи, есть опасения, что пожилые люди с деменцией могли быть в значительной степени исключены из таких ресурсов, как госпитализация и / или доступ к отделениям интенсивной терапии в пользу более молодых людей с меньшим количеством сопутствующих заболеваний. Это может частично объяснить высокий уровень смертности в этой популяции, 
особенно в учреждениях ухода и домах престарелых [66].

Более того, у пациентов с деменцией часто наблюдаются атипичные симптомы, такие как измененное психическое состояние (включая спутанность сознания, возбуждение, дезориентацию, отказ от помощи и потерю аппетита), как начальное проявление COVID-19, даже без лихорадки и кашля [64, 68], наряду с ухудшением исходного функционального статуса [9]. Таким образом, ожидание появления типичных респираторных симптомов приведет к отсрочке своевременной диагностики и лечения.

При лечении COVID-19 у пациентов, страдающих БА, следует учитывать лекарственные взаимодействия [69]. Уровни ингибиторов холинэстеразы могут увеличиваться во время лечения хлорохином, гидроксихлорохином и лопинавиром / ритонавиром из-за воздействия на цитохром Р450. Побочные эффекты со стороны сердца могут быть связаны как с азитромицином, так и с хлорохином, гидроксихлорохином, поэтому рекомендуется частый мониторинг электрокардиографии. Мемантин имеет низкий риск фармакокинетических взаимодействий и может быть более безопасной альтернативой при использовании лекарств для лечения COVID-19 у пациентов с БА. Нейролептики и антидепрессанты, часто используемые при деменции, потенциально могут взаимодействовать с азитромицином, хлорохином, гидроксихлорохином и лопинавиром / ритонавиром. Наконец, тоцилизумаб, рибавирин и фавипиравир не проявляют потенциального взаимодействия с лечением БА $[69,70]$.

Пандемия COVID-19 еще больше усугубляет уязвимость пожилых пациентов с когнитивными нарушениями, особенно тех, чей ежедневный уход зависит от семьи или опекунов. Это связано с увеличением заболеваемости и смертности, вызванными инфекцией, а также с косвенным воздействием пандемии на систему здравоохранения, от которой они зависят [71]. По данным зарубежных источников, люди с деменцией рискуют прекратить лечение во время изоляции, особенно те, кто зависит от внешней помощи [71].

Большинство смертей, связанных с COVID-19, произошло в учреждениях длительного ухода, где пациенты с деменцией составляют значительную часть и нуждаются в тесном контакте для оказания помощи в повседневном уходе [72,73]. Blackman et al. сообщили о быстром развитии COVID-19 в учреждении длительного ухода на 150 коек для людей с деменцией, где, несмотря на разработанные профилактические меры, в течение 3 недель после первого подтвержденного случая COVID-19 30 человек умерли и более 50 были подтвержденными случаями или имели симптомы, совместимые с этим заболеванием. Таким образом, крайне важно оборудовать такие учреж- дения соответствующими профилактическими мерами и возможностями быстрого обнаружения, чтобы избежать передачи и максимально защитить это и без того уязвимое население.

Многие центры за рубежом приняли меры и внедрили телемедицинские консультации для продолжения оказания помощи пациентам во время пандемии, как для запланированных последующих посещений, так и для срочных консультаций $[74,75,76]$. Такой постоянный уход во время пандемии был особенно важен для пациентов и лиц, осуществляющих уход, в более экстренной ситуации. С клинической точки зрения необходимо решить проблемы безопасности и правовые вопросы, связанные с конфиденциальностью и защитой данных, и некоторые тесты из нейропсихологических батарей пришлось адаптировать для выполнения их виртуальным способом [77]. После этого опыта вполне вероятно, что телемедицина продолжит играть важную роль в оценке пациентов с когнитивными нарушениями даже после сдерживания пандемии COVID-19.

Меры общества, принятые для замедления распространения COVID-19, вынудили к социальному дистанцированию и самоизоляции, и это могло способствовать возникновению поведенческих изменений у пациентов с когнитивными нарушениями [78]. Значительная часть пациентов с деменцией проживает в учреждениях для длительного ухода и в домах престарелых, которые во многих странах закрыты на карантин, что запрещает членам семьи посещать жителей или им выходить на улицу. Такие факторы, как социальная изоляция не следует недооценивать, поскольку было установлено, что они связаны с повышенным риском возникновения проблем со здоровьем, включая сердечно-сосудистые заболевания, депрессию или слабоумие $[79,80]$, и более высокий риск смертности, даже после учета соответствующих переменных, таких как состояние здоровья [81].

Исследование, проведенное отделением когнитивных расстройств в Испании, с участием 40 пациентов с легкой деменцией и легкими когнитивными нарушениями, посещающих программу когнитивной стимуляции, показало, что их психоневрологические симптомы значительно ухудшились после 5 недель изоляции (ведущими симптомами были апатия, тревога, возбуждение) [82]. Аналогичным образом, исследование о 139 пациентах с деменцией и субъективным снижением когнитивных функций из центра деменции в Риме показал ухудшение или начало поведенческих нарушений у 54,7\% (в основном возбуждение / агрессия, апатия и депрессия) после 1 месяца изоляции [78]. В 7,2\% случаев эти симптомы требовали корректировки или введения фармакологических методов лечения, в основном нейролептиков [78]. В выборке из 93 пожилых людей с когнитивными нарушениями или легкой деменци- 
ей те, кто живет один, сообщили о значительном ухудшении своего самочувствия, повышенном беспокойстве и проблемах со сном [83]. Кроме того, в исследовании с меньшей выборкой из 32 человек с лобно-височной долевой деменцией из центра лечения деменции в Триказе (Италия) лица, осуществляющие уход, были опрошены по телефону с использованием структурированной клинической оценки и сообщили, что по сравнению с их последним посещением (среднее значение 6,78 месяцев), 53\% пациентов показали значительное ухудшение когнитивной функции, особенно памяти, наряду с ухудшением поведения и языковой функцией во время изоляции по поводу COVID-19 [84].

Еще одна проблема - это влияние, которое пандемия может оказать на людей, находящихся на доклинической стадии деменции, или на тех, кто испытывает незначительные когнитивные изменения. Профилактика является наиболее важной стратегией в потенциально замедлении прогрессирования нейродегенеративных расстройств, и, учитывая повышенный риск негативных последствий для здоровья у пожилых людей, важно изучить и определить, может ли COVID-19 запускать или усугублять нейродегенеративные процессы в этой уязвимой группе.

Основные лаборатории, проводящие экспериментальные исследования в области болезни Альцгеймера, также пострадали от COVID-19, поскольку внезапная нехватка персонала из-за изоляции вынудила прекратить текущие эксперименты, что привело к потере ценных данных [85].

Пандемия COVID-19 вынудила приостановить большинство текущих клинических испытаний в области когнитивных нарушений на несколько месяцев, что безусловно повлияло на набор участников [86]. Это привело к значительным сбоям в управлении клиническими испытаниями, вызвав необходимость внесения поправок в протокол для устранения недостающих данных, отклонений, потери участников и разрешения использования телемедицины. В Соединенных Штатах Управление по санитарному надзору и качеству пищевых продуктов и медикаментов (FDA) выпустило 2 апреля 2020 документ, озаглавленный «Руководство по проведению клинических испытаний медицинских изделий во время пандемии COVID-19» (FDA, 2020 г.), призывая спонсоров руководствоваться здравым смыслом, чтобы принять решение о продолжении клинического испытания во время пандемии. Наконец, срыв клиниче- ских испытаний особенно повлиял на пациентов и их семьи в личном плане, поскольку они доверились фармакологическим исследованиям в борьбе с этим заболеванием, столкнулись с большой неуверенностью и беспомощностью в отношении своего будущего, но в целом стремились продолжить лечение, как только оно было безопасно [87].

Следует учитывать необходимость проведения ПЦР SARS-CoV-2 до начала лечения, как только участникам будет разрешено возобновить свое участие в клинических испытаниях, поскольку некоторые новые препараты, тестируемые против болезни Альцгеймера, обладают иммуноопосредованными механизмами [88].

\section{Зак^ючение}

Пандемия COVID-19 оказывает значительное влияние на многие аспекты здоровья, в том числе экономические и социальные аспекты во всем мире. Пожилое население, и особенно люди с сопутствующими заболеваниями, деменцией являются уязвимой группой, подверженной риску заражения этим заболеванием и имеющей более тяжелые формы и худшие исходы, включая смертность. На этом этапе крайне важно провести последующие обследования выживших после COVID-19 с использованием комплексных когнитивных и нейропсихиатрических оценок вместе с визуализацией мозга, если это необходимо, особенно для тех, кто перенес тяжелые формы заболевания на уровне отделения интенсивной терапии и интенсивной терапии для оценки неврологических проявлений в острой фазе. Необходимо выяснить отсроченные последствия и обеспечить поддержку психического здоровья и когнитивную реабилитацию, чтобы минимизировать потенциальные негативные последствия для психосоциального функционирования и качества жизни выживших. Учитывая повышенный риск негативных последствий для пожилых людей, важно изучить и определить, может ли COVID-19 вызвать или усугубить нейродегенеративные процессы в этой уязвимой группе, поскольку ранняя диагностика и вмешательство являются наиболее важными стратегиями замедления прогрессирования заболевания и нейродегенеративных расстройств. Важно отметить, что научные и клинические исследования, связанные с COVID-19, должны быть спланированы таким образом, чтобы обеспечить включение пожилых людей и возможности обеспечения их медикаментозной терапией.

\section{ЛИТЕРАТУРА}

1. Borges Do Nascimento I.J., Cacic N., Abdulazeem H.M., Von Groote T.C., Jayarajah U., et al. (2020). Novel Coronavirus infection (COVID-19) in humans: a scoping review and meta-analysis. J. Clin. Med. 9:941.

2. Wu Z., McGoogan J. M. (2020). Characteristics of and important lessons from the Coronavirus disease 2019 (COVID-19) outbreak in China: summary of a report of 72314 cases from the Chinese center for disease control and prevention. JAMA 323 1239-1242. 10.1001/jama.2020.2648 
3. Tsai S.T., Lu M.K., San S., Tsai C.H. (2020). The neurologic manifestations of Coronavirus disease 2019 pandemic: a systemic review. Front. Neurol. 11:498. 10.3389/ fneur.2020.00498

4. Ellul M.A., Benjamin L., Singh B., Lant S., Michael B.D., Easton A., et al. (2020). Neurological associations of COVID-19. Lancet Neurol. 19 767-783. 10.1016/S14744422(20)30221-0

5. Pinzon R.T., Wijaya V.O., Buana R.B., Al Jody A., Nunsio P.N. (2020). Neurologic characteristics in Coronavirus disease 2019 (COVID-19): a systematic review and metaanalysis. Front. Neurol. 11:565. 10.3389/fneur.2020.00565

6. Romero-Sanchez C.M., Diaz-Maroto I., Fernandez-Diaz E., Sanchez-Larsen A., Layos-Romero A., Garcia-Garcia J. (2020). Neurologic manifestations in hospitalized patients with COVID-19: the ALBACOVID registry. Neurology. 95 e1060-e1070. 10.1212/WNL.0000000000009937

7. Centers for Disease Control and Prevention (2020). COVIDView: A Weekly Surveillance Summary of US COVID-19 activity. Key updates for week, 29.

8. Lu L., Zhong W., Bian Z., Li Z., Zhang K., Liang B., et al. (2020). A comparison of mortality-related risk factors of COVID-19, SARS, and MERS: a systematic review and meta-analysis. J. Infect. 81 e18-e25. 10.1016/j.jinf.2020.07.002

9. Bianchetti A., Rozzini R., Guerini F., Boffelli S., Ranieri P., Minelli G., et al. (2020). Clinical presentation of COVID19 in Dementia patients. J. Nutr. Health Aging 24 560-562. 10.1007/s12603-020-1389-1 Lam M.H., Wing Y K., Yu M.W., Leung C.M., Ma R.C., Kong A.P., et al. (2009). Mental morbidities and chronic fatigue in severe acute respiratory syndrome survivors: Iong-term follow-up. Arch. Intern. Med. 169 2142-2147. 10.1001/archinternmed.2009.384

10. Dinakaran D., Manjunatha N., Naveen Kumar C., Suresh B.M. (2020). Neuropsychiatric aspects of COVID-19 pandemic: a selective review. Asian J. Psychiatr. $53: 102188$. 10.1016/j.ajp.2020.102188 Liguori C., Pierantozzi M., Spanetta M., Sarmati L., Cesta N., lannetta M. (2020). Subjective neurological symptoms frequently occur in patients with SARS-CoV2 infection. Brain Behav. Immun. 88 11-16. 10.1016/j.bbi.2020.05.037

11. Nalleballe K., Reddy Onteddu S., Sharma R., Dandu V., Brown A., Jasti M., et al. (2020). Spectrum of neuropsychiatric manifestations in COVID-19. Brain Behav. Immun. 88 71-74. 10.1016/j.bbi.2020.06.020

12. Rogers J.P., Chesney E., Oliver D., Pollak T.A., Mcguire P., Fusar-Poli P., et al. (2020). Psychiatric and neuropsychiatric presentations associated with severe coronavirus infections: a systematic review and meta-analysis with comparison to the COVID-19 pandemic. Lancet Psychiatry 7 611-627. 10.1016/s2215$0366(20) 30203-0$

13. Vindegaard N., Benros M.E. (2020). COVID-19 pandemic and mental health consequences: systematic review of the current evidence. Brain Behav. Immun. 10.1016/j. bbi.2020.05.048 [Epub ahead of print].

14. Varatharaj A., Thomas N., Ellul M.A., Davies N.W.S., Pollak T.A., Tenorio E.L. (2020). Neurological and neuropsychiatric complications of COVID-19 in 153 patients: a UK-wide surveillance study. Lancet Psychiatry 7 875-882. 10.1016/S2215-0366(20)30287-X

15. Parra A., Juanes A., Losada C.P., Alvarez-Sesmero S., Santana V.D., Marti I., et al. (2020). Psychotic symptoms in COVID-19 patients. A retrospective descriptive study. Psychiatry Res. 291:113254. 10.1016/j.psychres.2020.113254

16. Cipriani G., Danti S., Nuti A., Carlesi C., Lucetti C., Di Fiorino M. (2020). A complication of coronavirus disease 2019: delirium. Acta Neurol Belg. 120 927-932. 10.1007/ s13760-020-01401-7

17. Helms J., Kremer S., Merdji H., Clere-Jehl R., Schenck M., Kummerlen C., et al. (2020). Neurologic features in severe SARS-CoV-2 infection. N. Engl. J. Med. 382 2268-2270. 10.1056/nejmc2008597

18. Cai X., Hu X., Otte E.I., Wang J., An Y., Li Z., et al. (2020). Psychological distress and its correlates among COVID-19 survivors during early convalescence across age groups. Am.J. Geriatr. Psychiatry 28 1030-1039. 10.1016/j.jagp.2020.07.003

19. Yuan B., Li W., Liu H., Cai X., Song S., Zhao J. (2020). Correlation between immune response and self-reported depression during convalescence from COVID-19. Brain Behav. Immun. 88 39-43. 10.1016/j.bbi.2020.05.062

20. Zhang J., Lu H., Zeng H., Zhang S., Du Q., Jiang T., et al. (2020). The differential psychological distress of populations affected by the COVID-19 pandemic. Brain Behav. Immun. 87 49-50. 10.1016/j.bbi.2020.04.031

21. Batty G.D., Deary I.J., Luciano M., Altschul D.M., Kivimaki M., Gale C.R. (2020). Psychosocial factors and hospitalisations for COVID-19: prospective cohort study based on a community sample. Brain Behav. Immun. 10.1016/j.bbi.2020.06.021 [Epub ahead of print].

22. Pinna P., Grewal P., Hall J.P., Tavarez T., Dafer R.M., Garg R. (2020). Neurological manifestations and COVID-19: experiences from a tertiary care center at the Frontline. J. Neurol. Sci. 415:116969. 10.1016/j.jns.2020.116969

23. Chaumont H., San-Galli A., Martino F., Couratier C., Joguet G., Carles M., et al. (2020). Mixed central and peripheral nervous system disorders in severe SARS-CoV-2 infection. J. Neurol. 10.1007/s00415-020-09986-y [Epub ahead of print].

24. McLoughlin B.C., Miles A., Webb T.E., Knopp P., Eyres C., Fabbri A., et al. (2020). Functional and cognitive outcomes after COVID-19 delirium. Eur. Geriatr. Med. 10.1007/s41999-020-00353-8 [Epub ahead of print].

25. Troyer E.A., Kohn J.N., Hong S. (2020). Are we facing a crashing wave of neuropsychiatric sequelae of COVID-19? Neuropsychiatric symptoms and potential immunologic mechanisms. Brain Behav. Immun. 87 34-39. 10.1016/j.bbi.2020.04.027

26. Desai S.V., Law T.J., Needham D.M. (2011). Long-term complications of critical care. Crit. Care Med. 39371-379. 10.1097/ccm.0b013e3181fd66e5

27. Herridge M.S., Moss M., Hough C.L., Hopkins R.O., Rice T.W., Bienvenu 0.J., et al. (2016). Recovery and outcomes after the acute respiratory distress syndrome (ARDS) in patients and their family caregivers. Intensive Care Med. 42 725-738. 10.1007/s00134-016-4321-8

28. Marra A., Pandharipande P.P., Girard T.D., Patel M.B., Hughes C.G., Jackson J.C., et al. (2018). Co-occurrence of post-intensive care syndrome problems among 406 survivors of critical illness. Crit. Care Med. $461393-1401.10 .1097 / \mathrm{ccm} .0000000000003218$

29. Kim H.C., Yoo S.Y., Lee B.H., Lee S.H., Shin H.S. (2018). Psychiatric findings in suspected and confirmed middle east respiratory syndrome patients quarantined in hospital: a retrospective chart analysis. Psychiatry Investig. 15 355-360. 10.30773/pi.2017.10.25.1 
30. Sasannejad C., Ely E.W., Lahiri S. (2019). Long-term cognitive impairment after acute respiratory distress syndrome: a review of clinical impact and pathophysiological mechanisms. Crit. Care 23:352. Grasselli G., Zangrillo A., Zanella A., Antonelli M., Cabrini L., Castelli A. (2020). Baseline characteristics and outcomes of 1591 patients infected with SARS-CoV-2 admitted to ICUs of the Lombardy Region, Italy. JAMA 323 1574-1581. 10.1001/jama.2020.5394

31. Arentz M., Yim E., Klaff L., Lokhandwala S., Riedo F.X., Chong M., et al. (2020). Characteristics and outcomes of 21 critically ill patients with COVID-19 in Washington state. JAMA 323 1612-1614. 10.1001/jama.2020.4326

32. Hopkins R.0., Gale S.D., Weaver L.K. (2006). Brain atrophy and cognitive impairment in survivors of Acute Respiratory Distress Syndrome. Brain Inj. $20263-271$. $10.1080 / 02699050500488199$

33. Hopkins R.O., Weaver L.K., Collingridge D., Parkinson R.B., Chan K.J., Orme J.F., Jr. (2005). Two-year cognitive, emotional, and quality-of-life outcomes in acute respiratory distress syndrome. Am.J. Respir. Crit. Care Med. 171 340-347. 10.1164/rccm.200406-7630C

34. Heneka M.T., Golenbock D., Latz E., Morgan D., Brown R. (2020). Immediate and long-term consequences of COVID-19 infections for the development of neurological disease. Alzheimers Res. Ther. 12:69.

35. Ogier M., Andeol G., Sagui E., Bo G.D. (2020). How to detect and track chronic neurologic sequelae of Covid-19? Use of auditory brainstem responses and neuroimaging for long-term patient follow-up. Brain Behav. Immun. Health 5:100081. 10.1016/j.bbih.2020.100081

36. Zubair A.S., Mcalpine L.S., Gardin T., Farhadian S., Kuruvilla D.E., Spudich S. (2020). Neuropathogenesis and neurologic manifestations of the Coronaviruses in the age of Coronavirus disease 2019: a review. JAMA Neurol. 77 1018-1027. 10.1001/jamaneurol.2020.2065

37. Hoffmann M., Kleine-Weber H., Schroeder S., Kruger N., Herrler T., Erichsen S. (2020). SARS-CoV-2 cell entry depends on ACE2 and TMPRSS2 and is blocked by a clinically proven protease inhibitor. Cell 181 271-280.e8.

38. Xia H., Lazartigues E. (2008). Angiotensin-converting enzyme 2 in the brain: properties and future directions. J. Neurochem. 107 1482-1494. 10.1111/j.14714159.2008.05723.x

39. Netland J., Meyerholz D.K., Moore S., Cassell M., Perlman S. (2008). Severe acute respiratory syndrome coronavirus infection causes neuronal death in the absence of encephalitis in mice transgenic for human ACE2. J. Virol. 82 7264-7275. 10.1128/jvi.00737-08

40. Coolen T., Lolli V., Sadeghi N., Rovai A., Trotta N., Taccone F.S. (2020). Early postmortem brain MRI findings in COVID-19 non-survivors. Neurology 95 e2016-e2027. 10.1212/WNL.0000000000010116

41. Reichard R.R., Kashani K.B., Boire N.A., Constantopoulos E., Guo Y., Lucchinetti C.F. (2020). Neuropathology of COVID-19: a spectrum of vascular and acute disseminated encephalomyelitis (ADEM)-like pathology. Acta Neuropathol. 140 1-6. 10.1007/s00401-020-02166-2

42. Solomon I.H., Normandin E., Bhattacharyya S., Mukerji S.S., Keller K., Ali A.S., et al. (2020). Neuropathological features of Covid-19. N. Engl. J. Med. 383 989-992. 10.1056/NEJMc2019373

43. Puelles V.G., Lutgehetmann M., Lindenmeyer M.T., Sperhake J.P., Wong M.N., Allweiss L. (2020). Multiorgan and renal tropism of SARS-CoV-2. N. Engl. J. Med. 383 590-592. 10.1056/NEJMC2011400

44. Rita Egbert A., Cankurtaran S., Karpiak S. (2020). Brain abnormalities in COVID-19 acute/subacute phase: a rapid systematic review. Brain Behav. Immun. 10.1016/j. bbi.2020.07.014 [Epub ahead of print].

45. Frank-Cannon T.C., Alto L.T., Mcalpine F.E., Tansey M.G. (2009). Does neuroinflammation fan the flame in neurodegenerative diseases? Mol. Neurodegener. 4:47. 10.1186/1750-1326-4-47

46. Dantzer R. (2018). Neuroimmune interactions: from the brain to the immune system and vice versa. Physiol. Rev. 98 477-504. 10.1152/physrev.00039.2016 Rea I.M., Gibson D.S., Mcgilligan V., Mcnerlan S.E., Alexander H.D., Ross 0.A. (2018). Age and age-related diseases: role of inflammation triggers and cytokines. Front. Immunol. 9:586. 10.3389/fimmu.2018.00586

47. Chen G., Wu D., Guo W., Cao Y., Huang D., Wang H. (2020). Clinical and immunological features of severe and moderate coronavirus disease 2019. J. Clin. Invest. 130 2620-2629. 10.1172/jci137244

48. Huang C., Wang Y., Li X., Ren L., Zhao J., Hu Y. (2020). Clinical features of patients infected with 2019 novel coronavirus in Wuhan, China. Lancet 395 497-506.

49. Yang Y., Shen C., Li J., Yuan J., Wei J., Huang F. (2020). Plasma IP-10 and MCP-3 levels are highly associated with disease severity and predict the progression of COVID-19. J. Allergy Clin. Immunol. 146 119-127.e4.

50. Akiyama H., Barger S., Barnum S., Bradt B., Bauer J., Cole G.M., et al. (2000). Inflammation and Alzheimer's disease. Neurobiol. Aging $21383-421$.

51. Walker K.A., Gottesman R.F., Wu A., Knopman D.S., Gross A.L., Mosley T.H., et al. (2019). Systemic inflammation during midlife and cognitive change over 20 years: the ARIC Study. Neurology 92 e1256-e1267.

52. Sy M., Kitazawa M., Medeiros R., Whitman L., Cheng D., Lane T.E., et al. (2011). Inflammation induced by infection potentiates tau pathological features in transgenic mice. Am.J. Pathol. 178 2811-2822. 10.1016/j.ajpath.2011.02.012

53. Jacomy H., Fragoso G., Almazan G., Mushynski W.E., Talbot P.J. (2006). Human coronavirus OC43 infection induces chronic encephalitis leading to disabilities in BALB/C mice. Virology 349 335-346. 10.1016/j.virol.2006.01.049

54. Hosseini S., Wilk E., Michaelsen-Preusse K., Gerhauser I., Baumgartner W., Geffers R., et al. (2018). Long-term neuroinflammation induced by Influenza A virus infection and the impact on hippocampal neuron morphology and function. J. Neurosci. 38 3060-3080. 10.1523/jneurosci.1740-17.2018 Koenigsknecht-Talbo0 J., Landreth G.E. (2005). Microglial phagocytosis induced by fibrillar beta-amyloid and IgGs are differentially regulated by proinflammatory cytokines. J. Neurosci. 25 8240-8249. 10.1523/jneurosci.1808-05.2005

55. Kuo C.L., Pilling L.C., Atkins J.L., Masoli J.A.H., Delgado J., Kuchel G.A., et al. (2020). ApoE e4e4 genotype and mortality with COVID-19 in UK Biobank. J. Gerontol. A Biol. Sci. Med. Sci. 75 1801-1803. 10.1093/gerona/glaa169 
56. Kehoe P.G., Wong S., Al Mulhim N., Palmer L.E., Miners J.S. (2016). Angiotensin-converting enzyme 2 is reduced in Alzheimer's disease in association with increasing amyloid-beta and tau pathology. Alzheimers Res. Ther. 8:50.

57. Hwang J.M., Kim J.H., Park J.S., Chang M.C., Park D. (2020). Neurological diseases as mortality predictive factors for patients with COVID-19: a retrospective cohort study. Neurol. Sci. 41 2317-2324. 10.1007/s10072-020-04541-z

58. Miyashita S., Yamada T., Mikami T., Miyashita H., Chopra N., Rizk D. (2020). Impact of dementia on clinical outcomes in elderly patients with coronavirus 2019 (COVID-19): an experience in New York. Geriatr. Gerontol. Int. 20 732-734. 10.1111/ggi.13942

59. Suzuki M., Hotta M., Nagase A., Yamamoto Y., Hirakawa N., Satake Y. (2020). The behavioral pattern of patients with frontotemporal dementia during the COVID-19 pandemic. Int. Psychogeriatr. 10.1017/S104161022000109X [Epub ahead of print].

60. Williamson E.J., Walker A.J., Bhaskaran K., Bacon S., Bates C., Morton C.E. (2020). OpenSAFELY: factors associated with COVID-19 death in 17 million patients. Nature $584430-436$.

61. Cipriani G., Fiorino M.D. (2020). Access to care for dementia patients suffering from COVID-19. Am.J. Geriatr. Psychiatry 28 796-797. 10.1016/j.jagp.2020.04.009 Isaia G., Marinello R., Tibaldi V., Tamone C., Bo M. (2020). Atypical presentation of Covid-19 in an older adult with severe Alzheimer disease. Am.J. Geriatr. Psychiatry 28 790-791. 10.1016/j.jagp.2020.04.018

62. Ward C.F., Figiel G.S., Mcdonald W.M. (2020). Altered mental status as a novel initial clinical presentation for COVID-19 infection in the elderly. Am.J. Geriatr. Psychiatry 28 808-811. 10.1016/j.jagp.2020.05.013

63. Balli N., Kara E., Demirkan K. (2020). The another side of COVID-19 in Alzheimer's disease patients: drug-drug interactions. Int. J. Clin. Pract. $74:$ e13596.

64. University of Liverpool (2020). COVID-19 Drug Interactions.

65. Brown E.E., Kumar S., Rajji T.K., Pollock B.G., Mulsant B.H. (2020). Anticipating and mitigating the impact of the COVID-19 pandemic on Alzheimer's disease and related dementias. Am.J. Geriatr. Psychiatry 28 712-721. 10.1016/j.jagp.2020.04.010

66. Blackman C., Farber S., Feifer R.A., MorV., White E.M. (2020). An illustration of SARS-CoV-2 dissemination within a skilled nursing facility using heat maps. J. Am. Geriatr. Soc. 10.1111/jgs. 16642 [Epub ahead of print]. Cordasco F., Scalise C., Sacco M.A., Bonetta C.F., Zibetti A., Cacciatore G., et al. (2020). The silent deaths of the elderly in long-term care facilities during the Covid-19 pandemic: the role of forensic pathology. Med. Leg. J. 88 66-68. 10.1177/0025817220930552

67. Benaque A., Gurruchaga M.J., Abdelnour C., Hernandez I., Canabate P., Alegret M., et al. (2020). Dementia care in times of COVID-19: experience at Fundacio ACE in Barcelona. Spain. J. Alzheimers Dis. 76 33-40. 10.3233/jad-200547

68. Ousset P.J., Vellas B. (2020). Viewpoint: impact of the Covid-19 outbreak on the clinical and research activities of memory clinics: an Alzheimer's disease center facing the Covid-19 crisis. J. Prev. Alzheimers Dis. 7 197-198.

69. Padala S.P., Jendro A.M., Orr L.C. (2020). Facetime to reduce behavioral problems in a nursing home resident with Alzheimer's dementia during COVID-19. Psychiatry Res. 288:113028. 10.1016/j.psychres.2020.113028

70. Bilder R.M., Postal K.S., Barisa M., Aase D.M., Cullum C.M., Gillaspy S.R. (2020). InterOrganizational practice committee recommendations/guidance for teleneuropsychology (TeleNP) in response to the COVID-19 pandemic. Clin. Neuropsychol. 10.1080/13854046.2020.1767214 [Epub ahead of print].

71. Canevelli M., Valletta M., Toccaceli Blasi M., Remoli G., Sarti G., Nuti F., et al. (2020). Facing dementia during the COVID-19 outbreak. J. Am. Geriatr. Soc. 68 16731676. 10.1111/jgs. 16644

72. Holwerda T.J., Deeg D.J., Beekman A.T., Van Tilburg T.G., Stek M.L., Jonker C., et al. (2014). Feelings of loneliness, but not social isolation, predict dementia onset: results from the Amsterdam Study of the Elderly (AMSTEL). J. Neurol. Neurosurg. Psychiatry 85 135-142. 10.1136/jnnp-2012-302755

73. Courtin E., Knapp M. (2017). Social isolation, loneliness and health in old age: a scoping review. Health Soc. Care Community 25 799-812. 10.1111/hsc.12311

74. Holt-Lunstad J., Smith T.B., Baker M., Harris T., Stephenson D. (2015). Loneliness and social isolation as risk factors for mortality: a meta-analytic review. Perspect. Psychol. Sci. 10 227-237. 10.1177/1745691614568352

75. Lara B., Carnes A., Dakterzada F., Benitez I., Pinol-Ripoll G. (2020). Neuropsychiatric symptoms and quality of life in Spanish patients with Alzheimer's disease during the COVID-19 lockdown. Eur. J. Neurol. 10.1111/ene.14339

76. Goodman-Casanova J.M., Dura-Perez E., Guzman-Parra J., Cuesta-Vargas A., Mayoral-Cleries F. (2020). Telehealth home support during COVID-19 confinement for community-dwelling older adults with mild cognitive impairment or mild dementia: survey study. J. Med. Internet Res. 22: e19434. 10.2196/19434

77. Capozzo R., Zoccolella S., Frisullo M.E., Barone R., Dell'abate M.T., Barulli M.R., et al. (2020). Telemedicine for delivery of care in frontotemporal lobar degeneration during COVID-19 pandemic: results from Southern Italy. J. Alzheimers Dis. 76 481-489.

78. Bostanciklioglu M. (2020). Severe acute respiratory syndrome coronavirus 2 is penetrating to dementia research. Curr. Neurovasc. Res. 10.2174/156720261766620 0522220509 [Epub ahead of print].

79. Weinberg M.S., Patrick R.E., Schwab N.A., Owoyemi P., May R., Mcmanus A.J., et al. (2020). Clinical trials and tribulations in the COVID-19 era. Am.J. Geriatr. Psychiatry 28 913-920. 10.1016/j.jagp.2020.05.016

80. Geerts H., van der Graaf P.H. (2020). Salvaging CNS Clinical Trials Halted Due to COVID-19. CPT Pharmacometrics Syst. Pharmacol. $9367-370.10 .1002 / p s p 4.12535$

81. Perez-Grijalba V., Romero J., Pesini P., Sarasa L., Monleon I., San-Jose I. (2019). Plasma Abeta42/40 ratio detects early stages of Alzheimer's disease and correlates with CSF and neuroimaging biomarkers in the AB255 study. J. Prev. Alzheimers Dis. $634-41$. 